Published as:

Chatterjee, S., Moon, S., Hentschel, F., Gilmore, K., \& Seeberger, P. H. (2018). An Empirical Understanding of the Glycosylation Reaction. Journal of the American Chemical Society, 140(38), 11942-11953.

doi:10.1021/jacs.8b04525.

\title{
An Empirical Understanding of the Glycosylation Reaction
}

Sourav Chatterjee, Sooyeon Moon, Felix Hentschel, Kerry Gilmore, Peter Seeberger

\section{Variables Interrogated, Quantified}

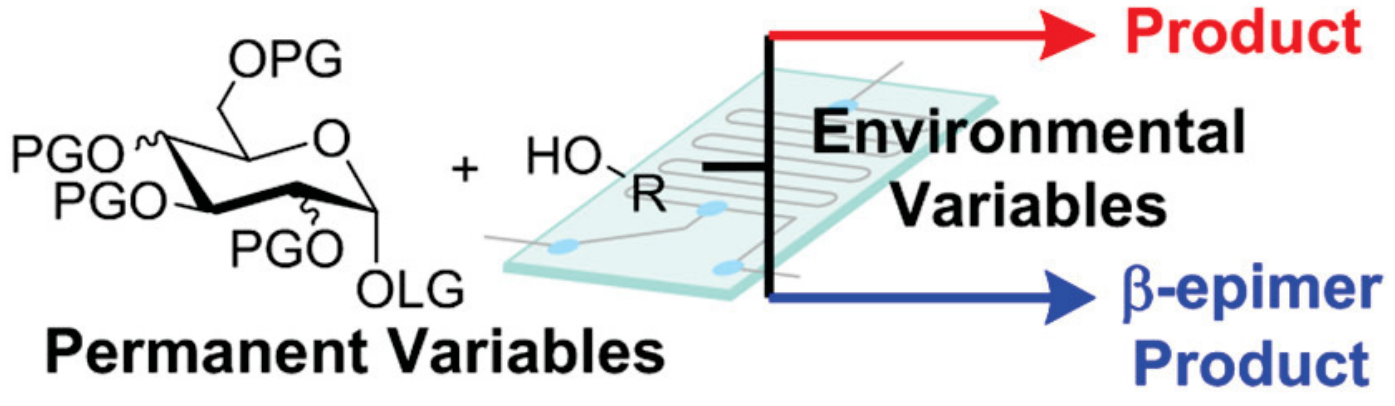

$$
\text { Automated Platform: } 270 \text { Experiments }
$$




\title{
An Empirical Understanding of the Glycosylation Reaction
}

\author{
Sourav Chatterjee, ${ }^{[\mathrm{a}] \dagger}$ Sooyeon Moon, ${ }^{[\mathrm{a}, \mathrm{b}] \dagger}$ Felix Hentschel, ${ }^{[\mathrm{a}]}$ Kerry Gilmore, ${ }^{*[\mathrm{a}]}$ Peter H. \\ Seeberger*[a,b] \\ a Department of Biomolecular Systems, Max-Planck-Institute of Colloids and Interfaces, Am \\ Mühlenberg 1, 14476 Potsdam, Germany \\ ${ }^{\mathrm{b}}$ Freie Universität Berlin, Institute of Chemistry and Biochemistry, Arnimallee 22, 14195 Berlin, \\ Germany
}

\begin{abstract}
:
Reliable glycosylation reactions that allow for the stereo- and regioselective installation of glycosidic linkages are paramount to the chemical synthesis of glycan chains. The stereoselectivity of glycosylations is exceedingly difficult to control due to the reaction's high degree of sensitivity and its shifting, simultaneous mechanistic pathways that are controlled by variables of unknown degree of influence, dominance, or interdependency. An automated platform was devised to quickly, reproducibly, and systematically screen glycosylations and thereby address this fundamental problem. Thirteen variables were investigated in as isolated a manner as possible, to identify and quantify inherent preferences of electrophilic glycosylating agents (donors) and nucleophiles (glycosyl acceptors). Ways to enhance, suppress, or even override these preferences using judicious environmental conditions were discovered. Glycosylations involving two specific partners can be tuned to produce either 11:1 selectivity of one stereoisomer or 9:1 of the other by merely changing the reaction conditions.
\end{abstract}

\section{Introduction}

Polysaccharides are a dominant class of biopolymers that play central roles in structure, energy storage, and biological functions. ${ }^{1}$ The chemical assembly of polysaccharides is conceptually simple as it relies on only a single type of bond forming reaction: the coupling of the electrophilic glycosylating agent ("glycosyl donor") with a nucleophile ("glycosyl acceptor"). Polysaccharides are more complex in their composition and structure than oligonucleotides or proteins. As such, the synthesis of glycans is very sensitive and relies on many diverse building blocks accounting for variants of hydroxyl substitution and stereochemistry on the pyran/furan core as well as multiple sites of connectivity for the creation of non-linear and branched structures. The number of possible structures is further significantly increased by the fact that each glycosidic bond generates a stereogenic center where two diastereomers (alpha or beta) can be formed (Figure 1).
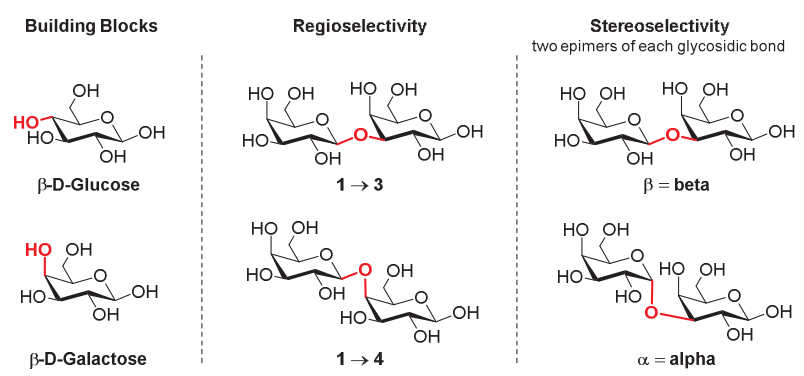

Figure 1: Three types of structural parameters contribute to the inherent complexity of glycans. Figure adapted from ref 2. 
The reaction mechanism of glycosidic bond formation is complex, often making controlled, selective bond formation elusive (Figure 2). ${ }^{3}$ The initial key step of the reaction is the cleavage of the bond between the anomeric carbon and the anomeric leaving group upon reaction with the activator. ${ }^{4}$ Thereby, a cationic ${ }^{5}$ intermediate is formed that can be engaged by the acceptor in a true $\mathrm{S}_{\mathrm{N}} 1$-type mechanism. The bond-forming reaction by attack of a nucleophile can either result in the formation of the more thermodynamically stable $\alpha$-product ${ }^{6}$ or via the potentially more accessible "top face" of the donor-intermediate to give the $\beta$-product. ${ }^{7}$ However, the carbocation can also form a close ion pair or a series of highly reactive glycoside intermediates from the reaction of part of the activator (conjugate base or byproduct). ${ }^{8}$ In these situations, the outcome of the glycosylation may resemble a $\mathrm{S}_{\mathrm{N}} 2$-type reaction. ${ }^{9}$ These intermediates - whose identity, relative ratios, kinetics, and favorabilities are essential to understand the mechanism - are often extremely difficult or even impossible to ascertain. ${ }^{10}$

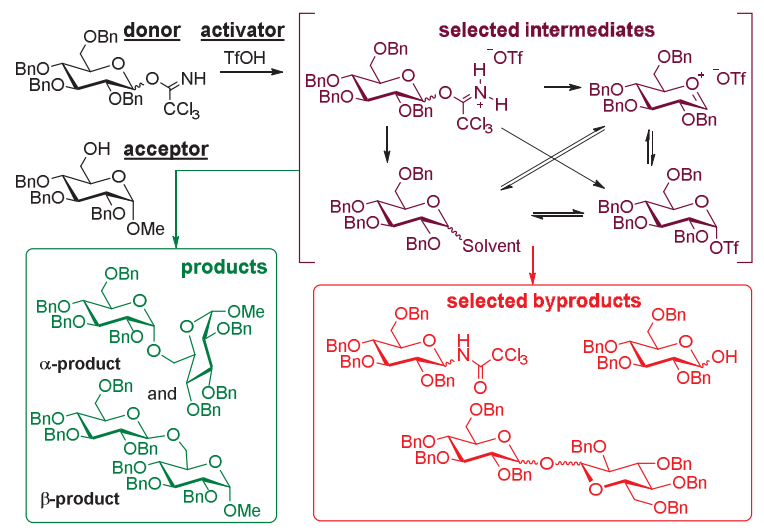

Figure 2: Glycosidic bond formation entails complex mechanistic pathways.

This is compounded by a lack of understanding regarding the underlying factors which have the potential to control the stereochemical outcome, both those dictated by the coupling partners (permanent) and the conditions chosen for the transformation (environmental, Figure 3). The donor ${ }^{11}$ contains five stereocenters, four of which can hyperconjugatively influence the activation as well as the stability and conformations of intermediates. ${ }^{12,13}$ Each of these four positions are also generally protected by groups capable of directly ${ }^{6,14}$ or indirectly influencing the reaction ${ }^{15}$ by donating ("arm") or withdrawing ("disarm") electrons from the glycosylating agent, ${ }^{16}$ or by restricting conformational freedom via bridged structures to influence reactivity ${ }^{17}$ and stereochemical outcome. ${ }^{18}$ The $\mathrm{C} 1$ leaving group chosen requires activation to induce cleavage, creating neutral or charged byproducts. ${ }^{19}$ With respect to the acceptor, the nucleophilicities of primary and secondary alcohols (the vast majority of glycosylations are C-O couplings) can depend on the orientation (axial/equatorial) of the alcohol, the adjacent protecting groups, ${ }^{20}$ and by structural changes induced by conformational locking ${ }^{21}$ or electronic modification. ${ }^{22}$ The sterics surrounding the nucleophile also will influence the rate of reaction and thus potentially the stereochemical outcome. 


\begin{tabular}{|c|c|c|c|}
\hline Permanent & \multicolumn{2}{|c|}{ Environmental } & Influence \\
\hline \multirow{2}{*}{$\begin{array}{l}\text { Leaving Group: } \\
\text { trichloroacetimidate } \\
\text { thioether, phosphate }\end{array}$} & $\frac{\text { Temperature: }}{-50 \rightarrow 70^{\circ} \mathrm{C}}$ & \multirow{2}{*}{$\begin{array}{l}\text { Activator: } \\
\text { TfOH, } \mathrm{MsOH}, \\
\mathrm{FSO}_{3} \mathrm{H},(\mathrm{Tf})_{2} \mathrm{NH}\end{array}$} & $\begin{array}{c}\text { Stabilities: } \\
\text { Conformers } \\
\text { Intermediates }\end{array}$ \\
\hline & \multirow{2}{*}{$\frac{\text { Concentration: }}{4.8 \rightarrow 20 \mathrm{mM}}$} & & Product(s) \\
\hline $\begin{array}{c}\text { Acceptor: } \\
\mathrm{MeOH}, \mathrm{EtOH}\end{array}$ & & \multirow{2}{*}{$\begin{array}{c}\text { Solvent: } \\
\text { DCM, ACN } \\
\text { Toluene, MTBE }\end{array}$} & $\begin{array}{l}\text { Activation: } \\
\text { Leaving Group }\end{array}$ \\
\hline $\begin{array}{c}\text { iPrOH, } t \mathrm{BuOH} \text {, } \\
\mathrm{di} / \text { trifluoroethanol }\end{array}$ & $\frac{\text { Residence Time: }}{45 \rightarrow 270 \mathrm{sec}}$ & & $\begin{array}{c}\text { Types of Intermediates } \\
\text { Reaction Pathways }\end{array}$ \\
\hline $\begin{array}{c}\text { Donor: } \\
\text { C2/C4: Glucose } \\
\text { Galactose, Mannose }\end{array}$ & $\frac{\text { Acceptor Equiv. }}{0.8 \rightarrow 10}$ & $\begin{array}{l}\text { Water: } \\
\text { Anhydrous } \\
\rightarrow 0.25 \text { equiv. }\end{array}$ & $\begin{array}{l}\text { Mechanism: } \\
\text { SN1- vs SN2-like } \\
\text { Nucleophilicity } \\
\text { Reaction Rates }\end{array}$ \\
\hline
\end{tabular}

Figure 3: A selection of the permanent and environmental factors of glycosylation reactions, with the specific examples examined in this study, as well as how these factors potentially influence the stereoselectivity.

The mechanistic path these coupling partners follow, as well as the intermediates which are formed, will be strongly influenced by the reaction environment. The physical properties of a solvent can promote bond cleavage, stabilize intermediates, and influence reaction pathways. The activator bears potential influencing factors resulting from interactions with the conjugate base, either via contact-ion pairs or covalent intermediates such as $\alpha$-triflates. ${ }^{10 a, 23}$ The temperature will impact the stability of intermediates, the reaction pathways followed, and product composition. Finally, traditional reaction parameters such as concentration, ${ }^{24}$ stoichiometry, ${ }^{25}$ reaction time, and mixing ${ }^{26}$ can have an impact on the efficiency of the reaction as well as the resulting stereoselectivity.

Carbohydrate chemistry has thus remained a conceptually simple but technically very demanding field, as optimization is required for every new reaction with strict maintenance of all reaction parameters. Additionally, the lack of understanding of what influence these factors have on stereochemical outcomes has left the selection of initial reaction parameters - such as the choice of anomeric leaving group, protecting groups, solvent, temperature, and activating agent often more of a matter of personal preference due to experience than knowledge-based decision making, leaving the field as much an art as a science.

In an effort to demystify glycosylation reactions and guide synthetic chemists towards optimal reaction conditions without prior reaction optimization, general guidelines regarding the selection of appropriate reaction conditions based on the intrinsic preferences of the coupling partners are urgently needed. However, before these can be determined, each of the factors influencing glcosylation reactions must first be identified and categorized before interrogatory experiments are devised and performed in a controlled, reproducible environment in as isolated a manner as possible. This reproducibility can be achieved using minimal amounts of material in microreactor flow chemistry setups. ${ }^{27}$ Herein, we describe the development and utilization of an automated microreactor/HPLC platform capable of varying reactant ratios, temperature, and reaction time autonomously. This instrument was used for the rapid and reproducible isolation and interrogation of variables influencing the stereoselectivity, providing an unprecedented, systematic, and quantifiable view of glysosidic bond formation from 270 experiments. The identification of, and factors providing control over, specific intermediates of the glycosylation reaction is outside the scope of this work.

\section{Instrument and Experimental Design}

The heart of the reaction optimizer platform is a $78 \mu \mathrm{L}$ silicon microreactor comprised of a separate addition port for donor, acceptor, and activator. ${ }^{28}$ Each of these lines is driven by two 
syringe pumps for reagent and solvent, allowing for both line purging and changes in concentration in any of the reagents (Figure 4). The combined solution passes through a mixing zone before entering the reaction zone. A quench is added prior to the reaction mixture exiting the chip to ensure the reaction is only occurring at the desired temperature. Once steady state is reached after ten reactor volumes, a sample is automatically removed via a $1 \mu \mathrm{L}$ HPLC injection loop. The temperature in the chip is maintained reliably within a range from -55 to $+70{ }^{\circ} \mathrm{C}$ within $\pm 0.1{ }^{\circ} \mathrm{C}$ by a surrounding aluminum block cooled or heated using a thermostat.

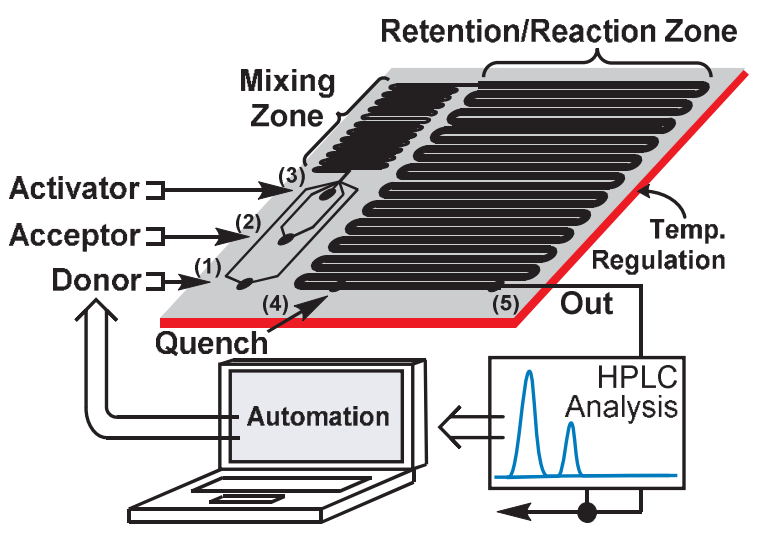

Figure 4: Automated glycosylation instrument consisting of three sections: multipart reaction section, HPLC analysis, and automation. For complete system details, see supporting information.

With automated system in hand, a model system was chosen to investigate the factors influencing the stereoselectivity of glycosylations (Figure 3). Three pyran cores - glucose, mannose, and galactose - were chosen as donors to probe the effects of the $\mathrm{C} 2$ and $\mathrm{C} 4$ positions, respectively. All hydroxyl groups, except at the anomeric position, were protected as nonparticipatory benzyl ethers. Three leaving groups - trichloroacetimidate, ethylthioether, and $n$ dibutylphosphate - were investigated. However, due to the similar stereochemical outcomes exhibited (vide infra), the more reactive glycosyl trichloroacetimidates were utilized primarily, activated with triflic acid rather than trimethylsilyltriflate (TMSOTf) to limit the potential reaction pathways. Methanol, ethanol, 2,2-difluoroethanol, 2,2,2-trifluoroethanol, isopropanol, and tert-butanol served as model acceptors to explore a range of steric and electronic effects. ${ }^{29}$ The temperatures investigated were limited by the reactivity of the donor and the physical properties of the solvents, kept under the respective boiling points.

The general glycosylation procedure required that the requisite benzyl protected donors be dried under high vacuum overnight prior to use and that all solvents, activators, and acceptors contained less than 3 ppm water. Glass syringes containing solutions of the donor $(50-110 \mathrm{mM})$, acceptor $(60-110 \mathrm{mM})$, and the activator $(22.6-120 \mathrm{mM})$ feed lines were diluted as desired via accompanying syringe pumps prior to mixing in the reactor. The reaction temperature, reagent concentration and stoichiometry, as well as the residence time were all set with the aid of the developed software. After reaction completion and inline quenching with pyridine, the reaction products were monitored by an automated injection into the online HPLC.

The results discussed below are based on those among the 270 glycosylation reactions that exhibited yields greater than $60 \%$ product (for a complete list, see the supporting information). The results of these investigations are discussed as isolated factors with respect to the change in temperature, starting with the permanent factors, followed by environmental. 


\section{Results and Discussion:}

Permanent: Donor Leaving Group (C1): Over the past century, a host of different leaving groups and corresponding activators to induce anomeric cleavage have been introduced as part of the quest for mild, selective, and high-yielding glycosylation reactions. ${ }^{19}$ To explore the effects these different groups have on the stereochemical outcome of glycosylation, a fully benzylated glucosyl $\alpha$-trichloroacetimidate (Schmidt donor), glucosyl $\beta$-ethanethio ether, or glucosyl $\alpha-n$ dibutylphosphate were reacted with isopropanol in DCM at temperatures ranging from $-50 \rightarrow$ $+30{ }^{\circ} \mathrm{C}$. The activation conditions for each leaving group were chosen to minimize differences in the conjugate bases/byproducts present in the solution, keeping triflate present in all cases. All other variables were kept constant.

Glycosylating agents with the three leaving groups gave nearly identical stereochemical outcomes under these conditions and differed mainly in conversion and yield at low temperatures (Figure 5). Glycosylation yields for thioglycosides dropped rapidly from $88 \%$ at $10{ }^{\circ} \mathrm{C}$ to $45 \%$ at $-10{ }^{\circ} \mathrm{C}$. A similar situation is observed for the phosphate $\left(60 \%\right.$ at $-10{ }^{\circ} \mathrm{C}$ to $30 \%$ at $\left.-30{ }^{\circ} \mathrm{C}\right)$. At these lower conversions, a slight increase $(<7 \%)$ in $\beta$-selectivity is observed as compared to the trichloroacetimidate donor (see SI).

Due to the similar stereochemical outcomes but increased reactivity range, the remaining studies were performed using the glycosyltrichloroacetimidates. Unexpectedly, stereoselectivity and temperature are related in a nearly linear fashion in DCM for many of the coupling partners examined. This linearity is lost, however, in certain environmental conditions (vide infra). Over the range examined $\left(-50{ }^{\circ} \mathrm{C}\right.$ to $\left.+30{ }^{\circ} \mathrm{C}\right)$, the selectivity of the glucose/isopropanol coupling ranges from favoring the $\beta$-product $(73 \%)$ to the $\alpha$-product $(39 \%)$. This corresponds to a temperature sensitivity (the slope of the plotted data) of $0.41 \%$ per ${ }^{\circ} \mathrm{C}$. These values serve as a comparison benchmark for all other variables examined herein. While the stereochemistry of some trichloroacetimidate donors, when reacted with TMSOTf, has been shown to have an influence on the stereochemical outcome of glycosylations, ${ }^{30}$ under our standard conditions in DCM, no difference was observed between the $\alpha$ - and $\beta$-glucose donor (Figure S17 in SI). ${ }^{31}$

Implication: The different leaving groups investigated, under the conditions explored, have little influence on the stereochemical outcome of glycosylations. The relative stabilities and desired reaction temperature are thus the key considerations when selecting the leaving group. The strongest influencing factor associated with the leaving group is the means of activation, particularly the conjugate base that is generated (vide infra). Coordination effects of the different neutral species formed upon decomposition of activated donor cannot be discounted, but appeared to be minimal under the conditions that were investigated. 


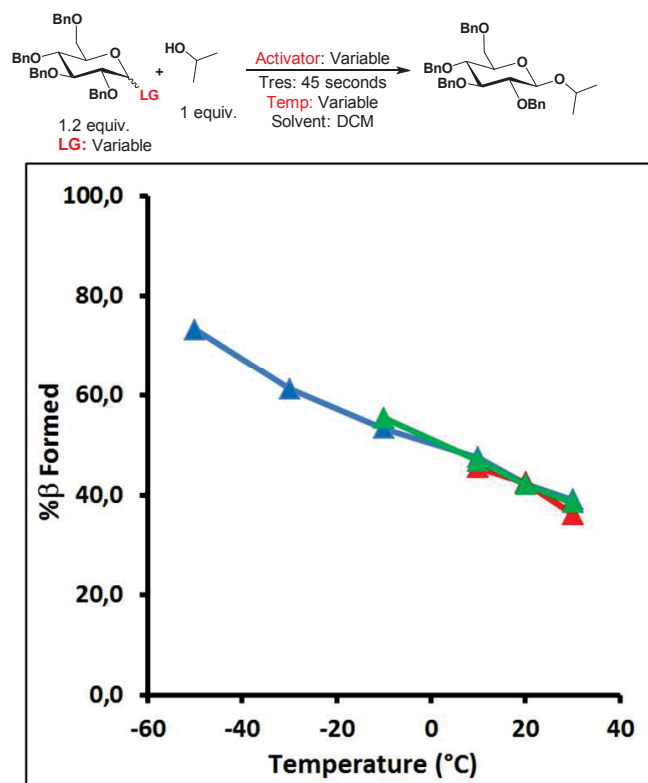

Figure 5: Comparison of stereoselectivities for glycosylations of glucose, bearing one of three leaving groups, with $i \mathrm{PrOH}$ as acceptor in DCM. For full experimental details, see SI. Figure code: Glucose $(\boldsymbol{\Delta})$; Trichloroacetimidate (blue) with $\mathrm{TfOH}(0.2$ equiv); ethyl thioether (red) with $\mathrm{TfOH}(0.2$ equiv) and $N$-iodosuccinamide (1.2 equiv); n-butylphosphate (green) with TMSOTf (1.2 equiv).

Donor Stereochemistry (C2 and C4): Donor activity and intermediate stability can both be influenced by through-bond or through-space hyperconjugation ${ }^{12}$ of the ether groups of the pyran core. $^{32}$ With non-participating groups, the influence of the $\mathrm{C} 2$ position has been shown to be significant in conformationally locked ${ }^{33}$ and unlocked glucose/mannose derivatives, ${ }^{34}$ as well as for less common derivatives such as glucose-/mannoseamine and the $\mathrm{C} 2$ fluorinated derivatives. ${ }^{35}$

Comparing the coupling of isopropanol with the $\alpha$-glucosyl and mannosyl trichloroacetimidates in DCM, a significant (34\%) decrease in temperature sensitivity is observed when the $\mathrm{C} 2$ benzyl ether is axial (mannose, Figure 6). The less sensitive mannose slightly favors the formation of the $\alpha$-product, with $\alpha: \beta$ ratios ranging from $48: 52\left(-50{ }^{\circ} \mathrm{C}\right)$ to $61: 39\left(30{ }^{\circ} \mathrm{C}\right)$.

Monosaccharides differing with respect to the $\mathrm{C} 4$ position, galactose (axial $\mathrm{C} 4$ ether) and glucose (equatorial $\mathrm{C} 4$ ether), exhibit similar temperature sensitivities $\left(\mathrm{T}_{\text {sens }}=0.43 \% /{ }^{\circ} \mathrm{C}\right.$ ) although galactose is 1.13 times more likely to give the $\beta$-product ( $9 \%$ more $\beta$-product formed) than glucose, ranging from $81 \% \beta$-selectivity at $-50{ }^{\circ} \mathrm{C}$ to $49 \%$ at $+30{ }^{\circ} \mathrm{C}$ (Figure 6).

Implication: Glycosylating agents display inherent preferences concerning mechanistic pathways and stereoselectivity. Glucose favors $\beta$-product formation, particularly at low temperatures, exhibiting a moderate degree of temperature sensitivity and follows a more $\mathrm{S}_{\mathrm{N}}$ 2-like reaction pathway. The preference for $\beta$-product formation is increased when the C4-ether is axial (galactose), and in DCM exhibits a similar $\mathrm{S}_{\mathrm{N}} 2$-like mechanism to glucose. The $\mathrm{C} 2$ position is significantly more influential, and mannose has an inherent preference for the formation of $\alpha$ product, proceeding via a more $\mathrm{S}_{\mathrm{N}} 1$-like pathway. These inherent preferences can be enhanced or overridden by the other reaction variables (vide infra). 


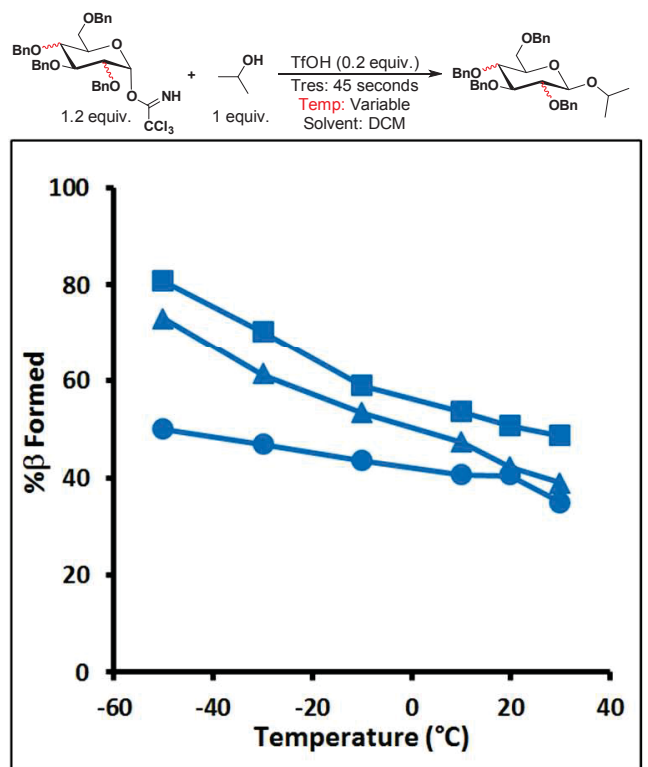

Figure 6: Comparison of the stereochemical outcome of three benzylated glycosyl trichloroacetimidates reacting with isopropanol and TfOH. For full experimental details, see SI.

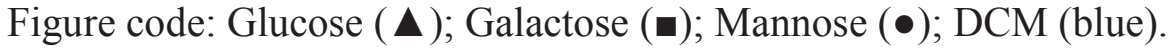

Acceptor Sterics and Electronics: To probe the factors that potentially influence the acceptor, a series of simple alcohols differing in both sterics ${ }^{36}$ and electronics (methanol, ethanol, isopropanol, tert-butanol, 2,2-difluoroethanol, 2,2,2-trifluoroethanol) were reacted with the glucosyl, galactosyl, and mannosyl trichloroacetmidate donors in DCM (Figure 7). Beginning with the "neutral" glucoside and the electronically "active" alcohols, a clear trend is observed in the series $\mathrm{t}-\mathrm{BuOH} / \mathrm{PrOH} / \mathrm{EtOH}$, with average stepwise increases of $8.2 \%$ and $6.7 \%$ in the $\beta$ selectivity at a given temperature, respectively, while a near identical rate of change with respect to temperature is maintained (Figure 7a). However, the least sterically encumbered alcohol, methanol, exhibits a $38 \%$ decrease in average temperature sensitivity as compared to ethanol ($0.3 \% \beta /{ }^{\circ} \mathrm{C}$ vs $-0.48 \% \beta /{ }^{\circ} \mathrm{C}$ ) and selectivities ranging from $71 \% \beta$ at $-50{ }^{\circ} \mathrm{C}$ to $47 \% \beta$ at $30{ }^{\circ} \mathrm{C}$.

The "active" acceptor series all exhibit higher $\beta$-selectivities with galactose compared with glucose, as was observed with isopropanol (Figure $7 \mathrm{~b}$ ). While the $\beta$-selectivity increases in the $t$ - $\mathrm{BuOH}$ to EtOH series $(4.2 \%$ and $4.8 \%)$, the temperature sensitivity is not constant as it is in the glucose series. With increasing sterics, the rate of change with respect to temperature decreases, from $-0.51 \% \beta /{ }^{\circ} \mathrm{C}(\mathrm{EtOH})$ to $-0.4 \% \beta /{ }^{\circ} \mathrm{C}(i \mathrm{PrOH})$ to $-0.27 \% /{ }^{\circ} \mathrm{C}(\mathrm{t}-\mathrm{BuOH})$. The coupling of galactosyl donor and methanol is more temperature sensitive than the rest of the series, with the $\beta$-selectivity ranging from $86 \%$ at $-50{ }^{\circ} \mathrm{C}$ to $41 \%$ at $30{ }^{\circ} \mathrm{C}\left(\mathrm{T}_{\text {sens }}=-0.56 \% \beta /{ }^{\circ} \mathrm{C}\right)$.

The coupling of mannose with isopropanol was much less temperature sensitive than for glucose and galactose (Figure 6). The stereoselectivity of mannose couplings is also much less sensitive to substitution of the acceptor (Figure 7c). Methanol, ethanol, and isopropanol all behave similarly with average selectivities ranging from $-51 \% \beta$ at $-50{ }^{\circ} \mathrm{C}$ to $37 \%$ at $30{ }^{\circ} \mathrm{C}$ (average $\mathrm{T}_{\text {sens }}=-0.17 \% \beta /{ }^{\circ} \mathrm{C}$ ) and only with the increased $t$-BuOH sterics was a deviation observed. $t$ - $\mathrm{BuOH}$ behaves similar to the other acceptors below $10{ }^{\circ} \mathrm{C}$, however with a stepwise decrease of $\sim 5 \%$ in $\beta$-selectivity. Above $10{ }^{\circ} \mathrm{C}$, a rapid change is observed, reaching $95 \%$ selectivity for the $\alpha$-product at $30{ }^{\circ} \mathrm{C}$.

Turning to the electronics of the acceptor, the insertion of electron-withdrawing fluorines beta to the nucleophilic oxygen has a pronounced effect on the stereoselectivity, favoring the formation of the $\alpha$-product (Figure 7d). When galactose ${ }^{37}$ was coupled to 2,2-difluoroethanol, the 
temperature sensitivity drops $70 \%$ compared to ethanol $\left(\mathrm{T}_{\text {sens }}=-0.15 \mathrm{vs}-0.51 \% \beta /{ }^{\circ} \mathrm{C}\right)$ and more $\alpha$ product is formed overall, with $\beta$ product formed ranging from $52 \%-50{ }^{\circ} \mathrm{C}$ to $41 \%$ at $30{ }^{\circ} \mathrm{C}$. Mannose, which already favors the formation of the $\alpha$-product, exhibits complete $\alpha$-selectivity when coupled with 2,2,2-trifluoroethanol.

Implication: A fine balance exists concerning the influential dominance between the inherent preferences of the donor and those of the acceptor. For donors favoring $\mathrm{S}_{\mathrm{N}} 2$-like pathways such as glucose and galactose, changes in acceptor nucleophilicity have a pronounced effect on the observed stereoselectivity, with stronger nucleophiles favoring the $\beta$-product at low temperatures with good levels of temperature sensitivity. The influence of the $\mathrm{C} 2$ position, favoring $\alpha$-product formation via an $\mathrm{S}_{\mathrm{N}}$ 1-type mechanism, overrides subtle differences in the nucleophile, and only major changes in nucleophilicity result in significant modifications to stereoselectivity. With a large decrease in the nucleophilicity $\left(\mathbf{C F}_{3} \mathrm{CH}_{2} \mathrm{OH}\right.$ vs $\left.\mathbf{C H}_{3} \mathrm{CH}_{2} \mathrm{OH}\right)$, the inherent favorability of mannose ( $\alpha$-formation), coupled with that of the low-nucleophilic acceptor, results exclusively in the $\alpha$-product. ${ }^{38}$
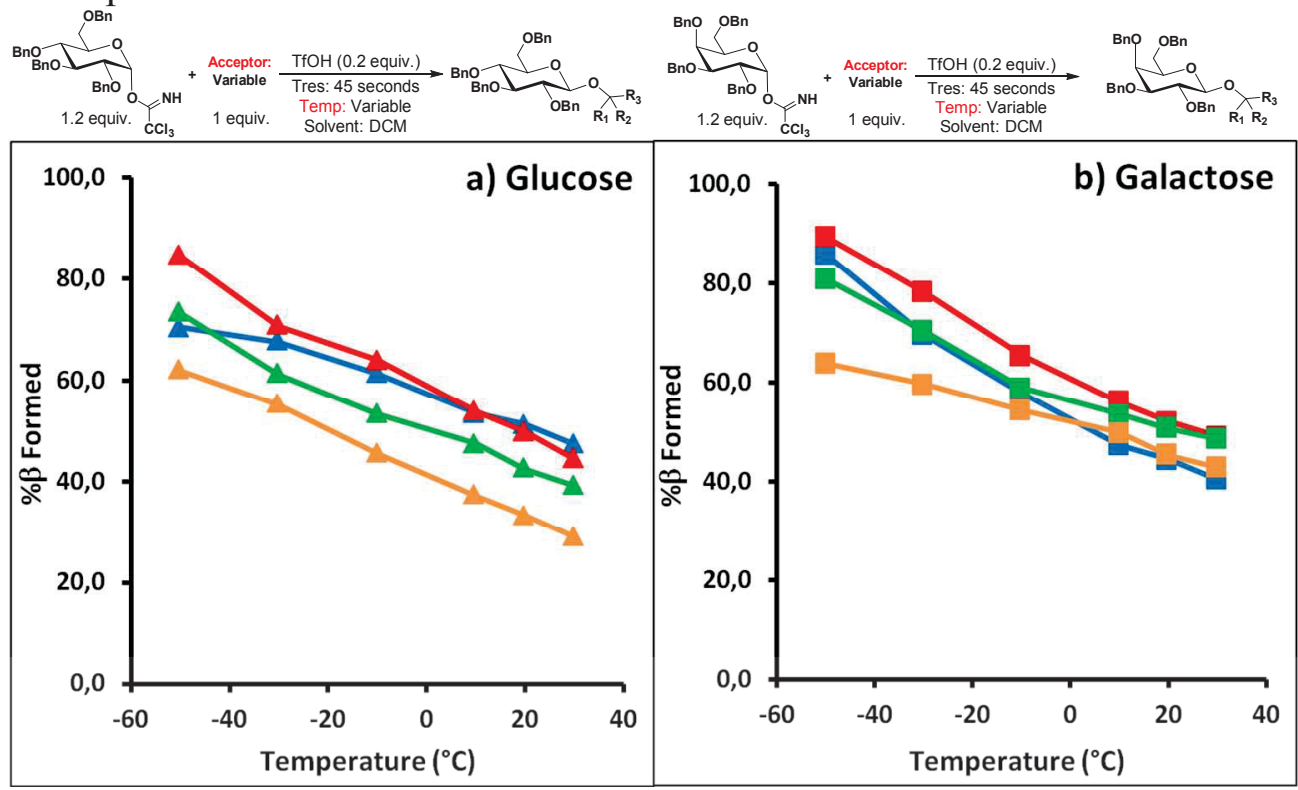


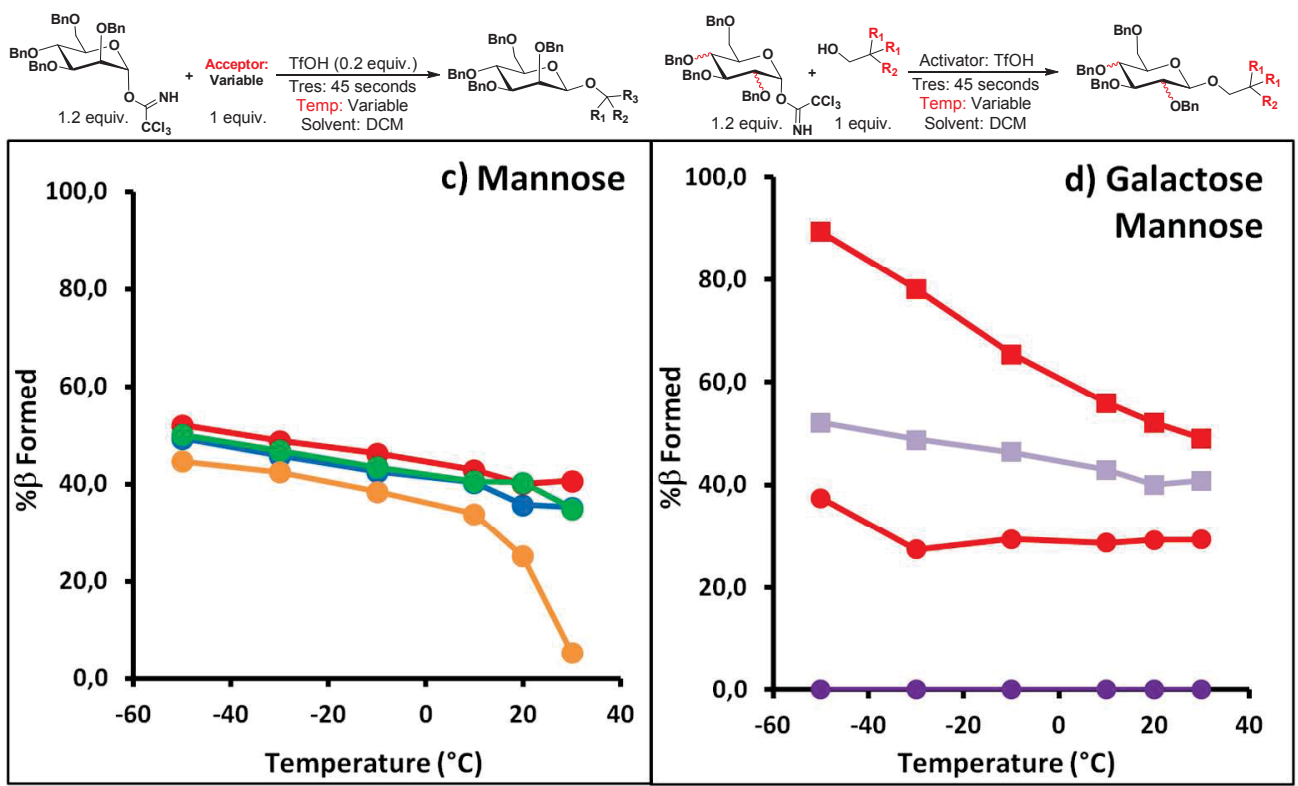

Figure 7: Comparison of different activators with three benzylated glycosyl trichloroacetimidates. For full experimental details, see SI. Figure code: Glucose $(\boldsymbol{\Delta})$; Galactose (匹); Mannose (•); $\mathrm{MeOH}$ (blue); $\mathrm{EtOH}$ (red); $i \mathrm{PrOH}$ (green); $t-\mathrm{BuOH}$ (orange); $\mathrm{CF}_{2} \mathrm{HCH}_{2} \mathrm{OH}$ (light purple, $\mathrm{R}_{1}=\mathrm{F}, \mathrm{R}_{2}=\mathrm{H}$ ); $\mathrm{CF}_{3} \mathrm{CH}_{2} \mathrm{OH}$ (dark purple, $\mathrm{R}_{1}=\mathrm{R}_{2}=\mathrm{F}$ ).

Environmental: The glycosylation conditions can enhance, diminish, or even override the intrinsic selectivities of a given donor/acceptor pair. Five factors were determined to strongly influence the reaction: temperature, stoichiometry, activator, presence of water, and the solvent. The other factors examined exhibited only minor influence (see SI for details).

Temperature: The strongest influence on $\alpha / \beta$ selectivity is temperature. At low temperatures, $\beta$ product formation is favored, presumably due to an increased proportion of $\mathrm{S}_{\mathrm{N}} 2$-like pathways from activated donors, favorable intermediate conformations, or species such as $\alpha$-triflates. The temperature influence can be overridden when the $\mathrm{S}_{\mathrm{N}} 1$ pathway becomes dominant.

Implication: The degree of variance observed in the stereoselective outcome of a glycosylation as a function of temperature ${ }^{28}$ emphasizes the need for precise temperature control. Temperature variation provides the most straightforward means of modifying the stereoselectivity. The clear temperature dependency breaks down in other solvents, presenting distinct mechanistic zones (vide infra).

Stoichiometry: Reagent stoichiometry should matter for processes that involve $\mathrm{S}_{\mathrm{N}} 2$-like mechanisms. Often, the donor is used in excess in a glycosylation ${ }^{39}$ to ensure high yields when poor nucleophiles are used or when other side reactions consume the activated donor. However, additional equivalents of acceptor can influence not only yield, but also selectivity. ${ }^{25}$

To investigate the effect of acceptor stoichiometry on stereoselective outcome, the perbenzylated galactosyl trichloroacetimidate was reacted with the poorly nucleophilic 2,2difluoroethanol. With only one equivalent acceptor, the process is temperature independent, favoring $\alpha$-formation $(73 \%)$. At $30^{\circ} \mathrm{C}$, little change is observed when five or ten equivalents acceptor are added. At lower temperatures, however, increased amounts of acceptor result in an increased amount of the $\beta$-product formed (at a rate of 0.15 and $0.22 \% \beta /{ }^{\circ} \mathrm{C}$ ) resulting in increases 
from $30 \% \beta$-product ( 1 equiv.) to $42 \%$ and $50 \% \beta$-diastereomer for one and ten equivalents, respectively at $-50{ }^{\circ} \mathrm{C}$ (Figure 8 , left).

This effect is not dominant. While the stoichiometry of the acceptor has been shown to affect the selectivity in competition experiments with conformationally locked mannose donors, ${ }^{29}$ the perbenzylated mannosyl trichloroacetimidate exhibited little difference with one and five equivalents of isopropanol in toluene, a $4.4 \%$ increase in $\beta$-product at elevated temperatures (Figure 8b).

Implication: For donors with weaker inherent preferences such as glucose and galactose that favor the $\mathrm{S}_{\mathrm{N}} 2$ pathway, the formation of $\beta$-product can be enhanced by increasing the equivalents of the acceptor. Marked increases can be seen even for poor nucleophiles, and larger differences are expected for stronger nucleophiles: a $26 \%$ increase in the $\beta$-selectivity is observed when ten equivalents of methanol were reacted with galactosyl trichloroacetimidate at $30{ }^{\circ} \mathrm{C}$ as compared to the 0.8 equivalents used in our standard experiments (see SI). However, the strongly influencing axial C2 group in mannose overrides this factor, resulting in little difference between one and five equivalents of isopropanol over a wide temperature range.

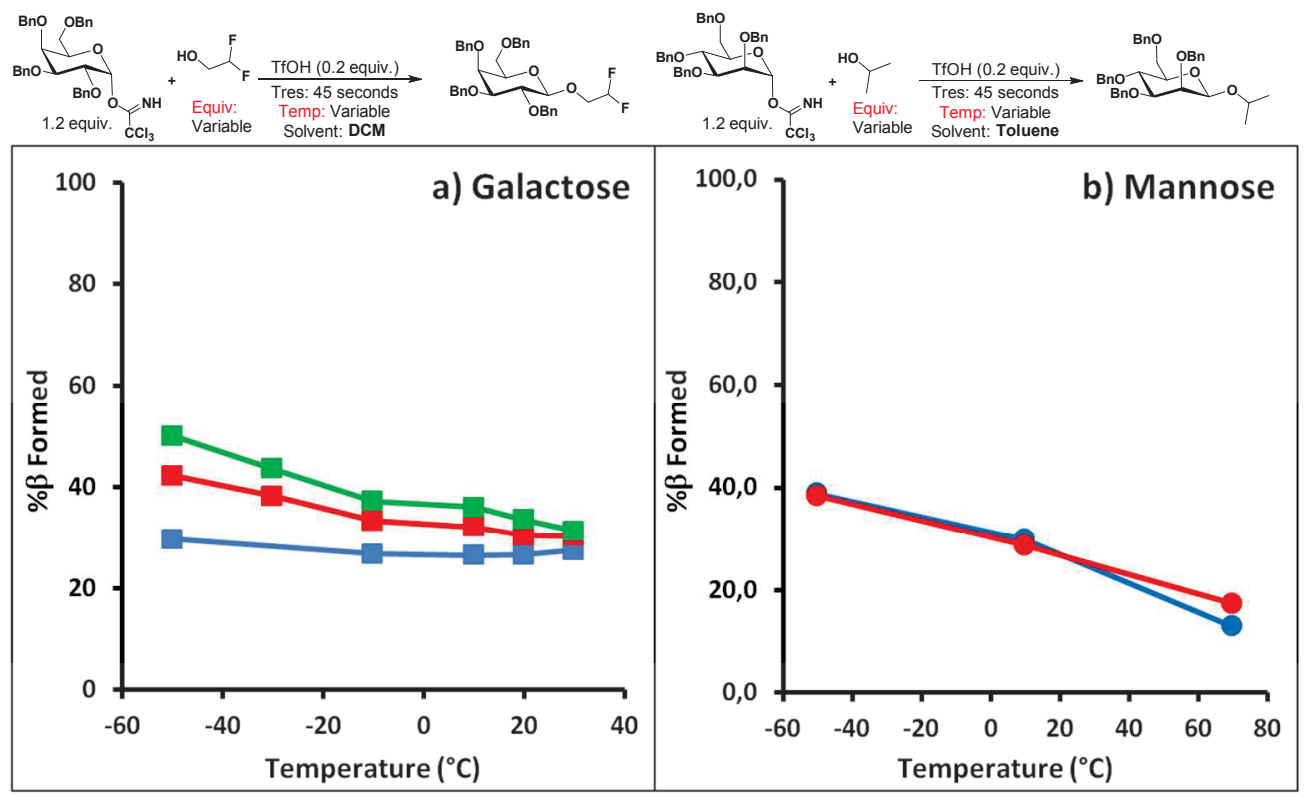

Figure 8: Comparison of acceptor stoichiometry in the reaction of galactosyl trichloroacetimidate in DCM and the mannosyl trichloroacetimidate in toluene. For full experimental details, see SI. Figure code: Galactose (匹); Mannose (•); Donor:Acceptor 1:1 (blue); 1:5 (red); 1:10 (green). For mannose, the ratio of Donor:Acceptor in the blue line is 1:0.8.

Activator: The activator has multiple potential roles in a glycosylation: the native species activates the leaving group, while the conjugate base stabilizes charged intermediates and may even trap these intermediates by reversible covalent bond formation. To investigate what influence the activator, and in particular the conjugate base of the activator, exerts on stereoselectivity, a range of substoichiometric acids were reacted with perbenzylated mannose and glucose donors using tert-butanol as acceptor in DCM. When triflic acid (blue) ${ }^{40}$ is exchanged for fluorosulfuric (green) or methanesulfuric acid (orange), a loss in temperature sensitivity is observed with mannose, giving an approximate 3:2 ratio of $\alpha$ : $\beta$ (Figure 9a). Alternatively, when the less electron-rich triflimide $\left(\mathrm{Tf}_{2} \mathrm{NH}\right)$ is used, a significant change is 
observed. Here, temperature sensitivity is observed at lower temperatures (approx. $-10{ }^{\circ} \mathrm{C}$ vs. +10 ${ }^{\circ} \mathrm{C}$ with $\mathrm{TfOH}$ ) and quickly approaches near complete $\alpha$-selectivity above $+10{ }^{\circ} \mathrm{C}$. This dataset represents the most rapid change of stereoselectivity with respect to temperature of all conditions examined. Interestingly, this finding differs from previous observations for the conformationally locked mannosyl trichloroacetimidate, which favored $\beta$-product formation using $\mathrm{Tf}_{2} \mathrm{NH}$ as activator. $^{41}$

In glucose the situation is different. With $\mathrm{TfOH}$, tert-butanol exhibits poor selectivity ranging from $60 \% \beta$-product at $-50{ }^{\circ} \mathrm{C}$ to $40 \%$ at $30{ }^{\circ} \mathrm{C}$. When the activator is changed to $\mathrm{Tf}_{2} \mathrm{NH}$, the reaction is almost completely $\beta$-selective $(95 \%)$ at $-50{ }^{\circ} \mathrm{C}$ and is nearly 1.5 times more sensitive to temperature (Figure $9 b$ ).

Implication: Through modification of the nucleophilicity of the conjugate base of the acceptor, the inherent stereoselective preference of the donor can be enhanced. Thus, by changing from $\mathrm{TfOH}$ to $\mathrm{Tf}_{2} \mathrm{NH}$ - both equally capable of activating the trichloroacetimidate donor - the mannose donor gives more of the $\alpha$-product and breaks way from the $\mathrm{S}_{\mathrm{N}} 1$-like pathway at lower temperatures. For glucose, the same change in acceptor sees a large increase in $\beta$-product formation and increased $\mathrm{S}_{\mathrm{N}}$ 2-like behavior. This factor appears to have a high degree of dominance in glycosylation reactions.
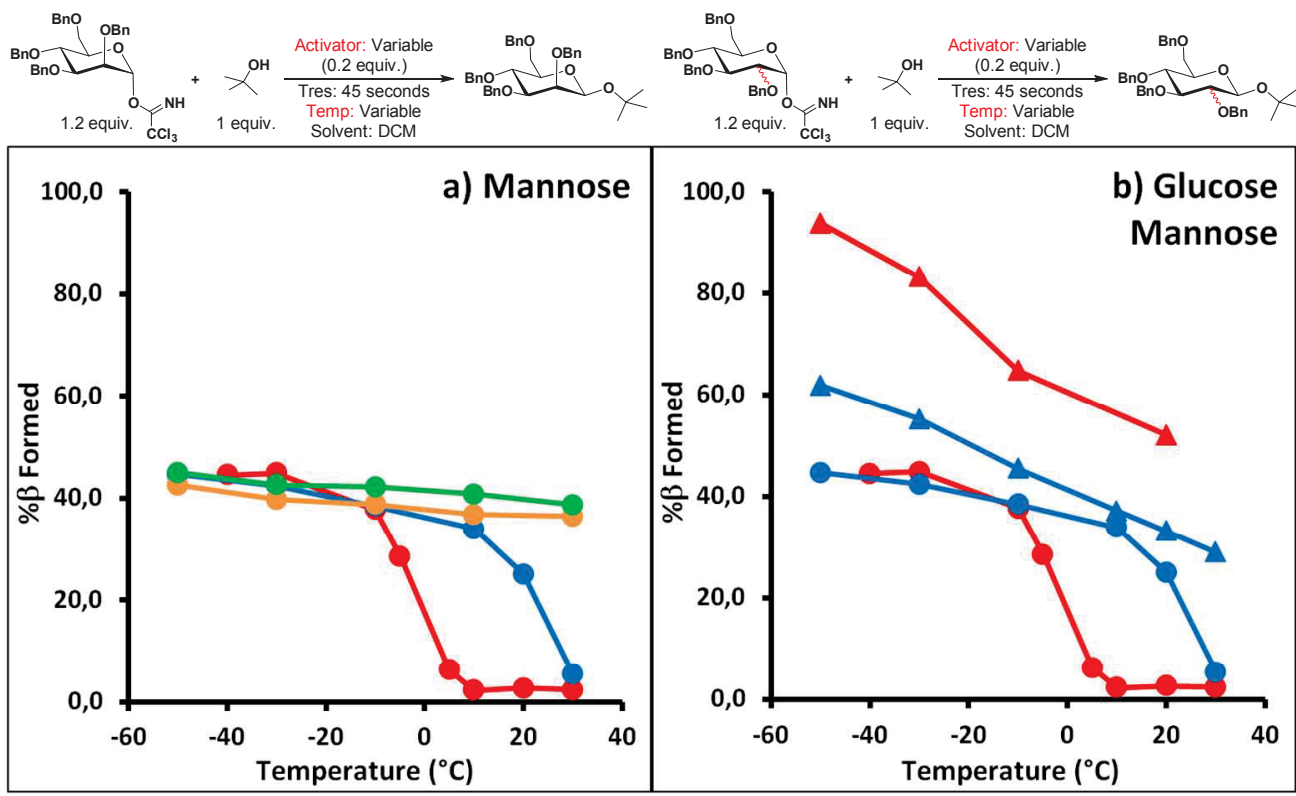

Figure 9: a) Comparison of different activators on the coupling of mannosyl trichloroacetimidate with $t$-BuOH. b) $\mathrm{Tf}_{2} \mathrm{NH}$ enhances the inherent stereoselective favorabilities of donors. For full

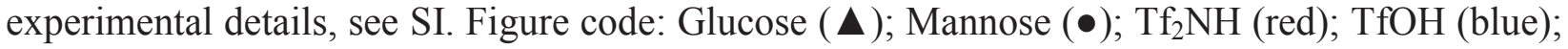
$\mathrm{MsOH}$ (orange); $\mathrm{FSO}_{3} \mathrm{H}$ (green).

Presence of Water: Water is detrimental to glycosylation reactions in organic solvents, as it efficiently traps the activated donor and/or intermediates to give the hydrolyzed donor, which cannot be reactivated under standard glycosylation conditions. Here, all solvents were dried to less than 3 ppm water (see SI) to minimize this unproductive pathway.

However, it was found that even substoichiometric amounts of water can have a significant influence not on the yield, but rather on the stereoselectivity of glycosylations. Under the standard "anhydrous" conditions, the coupling of mannose and tert-butanol exhibits low temperature sensitivity until $10^{\circ} \mathrm{C}$, when a rapid change is observed until almost complete $\alpha$ - 
selectivity is achieved at $30{ }^{\circ} \mathrm{C}$ (Figure 10). However, in the presence of 0.25 equivalents of water, the amount present in the solid glycosylating agent prior to the extensive drying procedure, a complete loss of temperature sensitivity is observed, and the reaction follows a $S_{N} 1$-like path over the entire temperature range. No difference was observed in the coupling of glucose and isopropanol under "anhydrous" conditions or in the presence of 0.25 equivalents of water. The yields for the two respective coupling conditions for mannose and glucose couplings were similar.

Implication: High percentages of water present in the glycosylation medium results in the competitive trapping of intermediates to give the hydrated donor byproduct. However, in substoichiometric amounts, water promotes the $\mathrm{S}_{\mathrm{N}} 1$ pathway without significant increases in byproduct formation. As such, donor/acceptor pairs which favor $\mathrm{S}_{\mathrm{N}}$ 1-like processes, such as mannose or electron-deficient acceptors, will be affected by small amounts of water in the medium, while coupling partners favoring $\mathrm{S}_{\mathrm{N}} 2$-like pathways will be less affected.

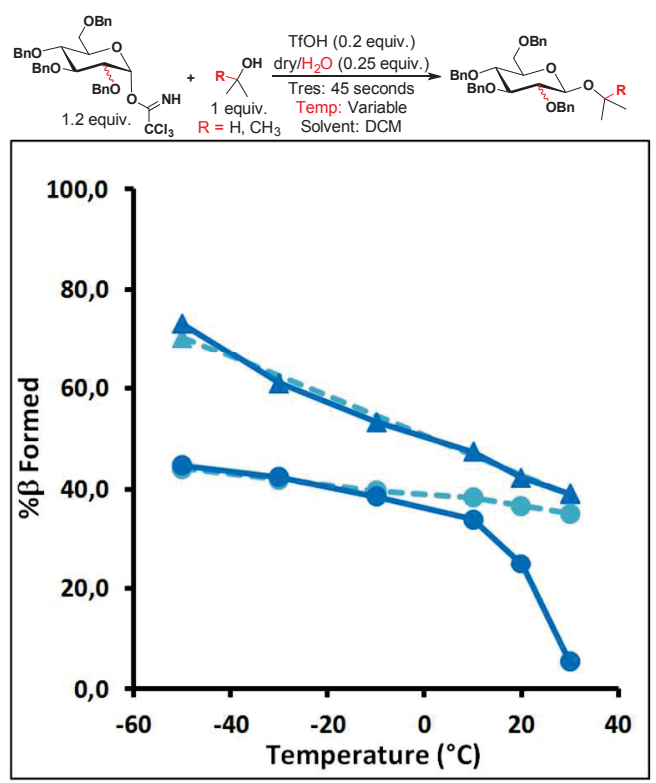

Figure 10: Comparison of stereoselectivities of glucosyl trichloroacetimidate with $i \mathrm{PrOH}$ and mannosyl trichloroacetimidate with $t-\mathrm{BuOH}$ under "anhydrous" conditions and in the presence of substoichiometric amounts of water. For full experimental details, see SI. Figure code: Glucose $(\boldsymbol{\Delta})$; Mannose (•); DCM (blue); DCM + 0.25 equiv. $\mathrm{H}_{2} \mathrm{O}$ (light blue, dashed line).

Solvent: The choice of solvent used for glycosylation reactions greatly influences the stereochemical reaction outcome, ${ }^{42}$ which can result from solvent coordination or conformer and counterion distribution. ${ }^{43}$ A general observation is that $\alpha$-linkages form preferentially in ether solvents, while $\beta$-linkages are formed in acetonitrile. To investigate how the different solvents enhance, suppress, or override the other factors influencing glycosylation stereoselectivity, four solvents were examined: dichloromethane (DCM), acetonitrile (ACN), toluene (Tol), and methyl tert-butyl ether (MTBE).

Glucose, which bears no inherent influencing groups, is strongly affected by the solvent choice (Figure 11, top). Compared to the "standard" solvent DCM, a significant increase in the formation of $\beta$-product is observed with acetonitrile when using isopropanol as acceptor, ranging from $90 \% \beta$ at $-30{ }^{\circ} \mathrm{C}$ to $58 \% \beta$ at $70{ }^{\circ} \mathrm{C}$. The coupling also exhibits a $27 \%$ decrease in temperature 
sensitivity versus DCM. While toluene gives a very similar range of selectivities to DCM (81\% $\rightarrow 38 \%$ vs $73 \% \rightarrow 39 \%$ ), two distinct mechanistic pathways appear to be occurring above and below $10{ }^{\circ} \mathrm{C}$, with $\mathrm{S}_{\mathrm{N}}$ 2-like favored at lower temperatures and $\mathrm{S}_{\mathrm{N}}$ 1-like at higher temperatures. The largest change in stereoselectivity is observed with MTBE. Here, a relatively temperatureinsensitive process greatly favors $\alpha$-product formation $\left(15 \% \beta\right.$ at $-50{ }^{\circ} \mathrm{C} \rightarrow 18 \% \beta$ at $\left.+50{ }^{\circ} \mathrm{C}\right)$. The average selectivity across all solvents and temperatures examined for the coupling of perbenzylated glucose donor and isopropanol using $\mathrm{TfOH}$ as activator is nearly equal mixture of diastereomers $(52: 48 \alpha: \beta)$.

Compared to glucose, the axial C4-O-benzyl ether of galactose increases the inherent preference for the formation of the $\beta$-diastereomer via a $S_{N} 2$-like pathway, as is reflected across all solvents examined (Figure 11, middle). Again, acetonitrile enhances this preference, giving higher $\beta$-selectivities with decreased temperature sensitivities (50\% decrease compared to DCM). In toluene, the coupling of galactose and isopropanol gives a broad range of selectivities, from $90 \% \beta$ at $-50{ }^{\circ} \mathrm{C}$ to only $31 \% \beta$ at $70{ }^{\circ} \mathrm{C}$. Similar to glucose, a selectivity plateau is observed in toluene as well, albeit at higher temperatures $\left(>50^{\circ} \mathrm{C}\right)$. While the $\alpha$-product is formed predominantly also in MTBE, the change is not as pronounced as with glucose, and the system exhibits a similar temperature sensitivity as observed in DCM. The average selectivity across all solvents and temperatures examined for the coupling of perbenzylated galactose donor and isopropanol using $\mathrm{TfOH}$ as activator is 43:57 ( $\alpha: \beta), 1.2$ times more selective for the $\beta$-product compared to glucose.

Mannose has a strong inherent preference to form the $\alpha$-product, and this effect is enhanced in all solvents examined compared to DCM. Near stepwise increases in $\alpha$-product selectivity by $10 \%$ are seen in the DCM/toluene/MTBE progression. Similarly low temperature sensitivities are observed with these solvents, with a slight increase in $\alpha$-selectivity observed in toluene at higher temperatures $\left(>50^{\circ} \mathrm{C}\right)$. Acetonitrile, while acting to enhance the inherent preference of the donor, appears to proceed via two distinct mechanisms based on temperature similar to what is observed with glucose/galactose in toluene. At lower temperatures, the selectivity is constant at $35 \% \beta$-product formation. Above $10{ }^{\circ} \mathrm{C}$, a rapid increase in temperature sensitivity $\left(1.1 \% /{ }^{\circ} \mathrm{C}\right)$ is observed as compared to the low temperature data, reaching near complete $\alpha$-selectivity above $30{ }^{\circ} \mathrm{C}$. The average selectivity across all solvents and temperatures examined for the coupling of perbenzylated mannose donor and isopropanol using $\mathrm{TfOH}$ as activator is 73:27 ( $\alpha: \beta), 1.4$ times more selective for the $\alpha$-product compared to glucose.

Implication: The solvent has a strong effect on the resulting $\alpha / \beta$-selectivity of glycosylations. Acetonitrile enhances the inherent donor preferences, providing higher proportions of the $\beta$ product for glucose/galactose and $\alpha$-product for mannose. Toluene also enhances this favorability, though to a lesser extent. Methyl tert-butyl ether enhances the formation of the $\alpha$ product for all donors. Glucose is particularly susceptible; however the major diastereomer is the $\alpha$-product for galactose at temperatures above $-40{ }^{\circ} \mathrm{C}$. Mannose is less strongly influenced, but does exhibit increased proportions of the $\alpha$-diastereomer in MTBE.

Certain solvents also appear able to exhibit distinct mechanisms within certain temperature zones, as evidenced by the sometimes sharp inflection points in the plotted data. In acetonitrile, the coupling of mannose and isopropanol undergoes a shift between $0{ }^{\circ} \mathrm{C}$ and $20{ }^{\circ} \mathrm{C}$, rapidly changing from $\mathrm{S}_{\mathrm{N}}$ 1-like to $\mathrm{S}_{\mathrm{N}}$ 2-like to give near complete selectivity for the $\alpha$-product. In toluene, glucose exhibits a similar mechanistic shift from $\mathrm{S}_{\mathrm{N}}$ 2-like to $\mathrm{S}_{\mathrm{N}} 1$-like at $20{ }^{\circ} \mathrm{C}$ and galactose at $50{ }^{\circ} \mathrm{C}$. Interestingly, the $\mathrm{S}_{\mathrm{N}}$ l-like plateau of stereoselectivity occurs in the same region for all three donors $(65: 35 \alpha: \beta$, Figure 11d) in the two different solvents, potentially providing a general value for couplings proceeding via this mechanistic path. 


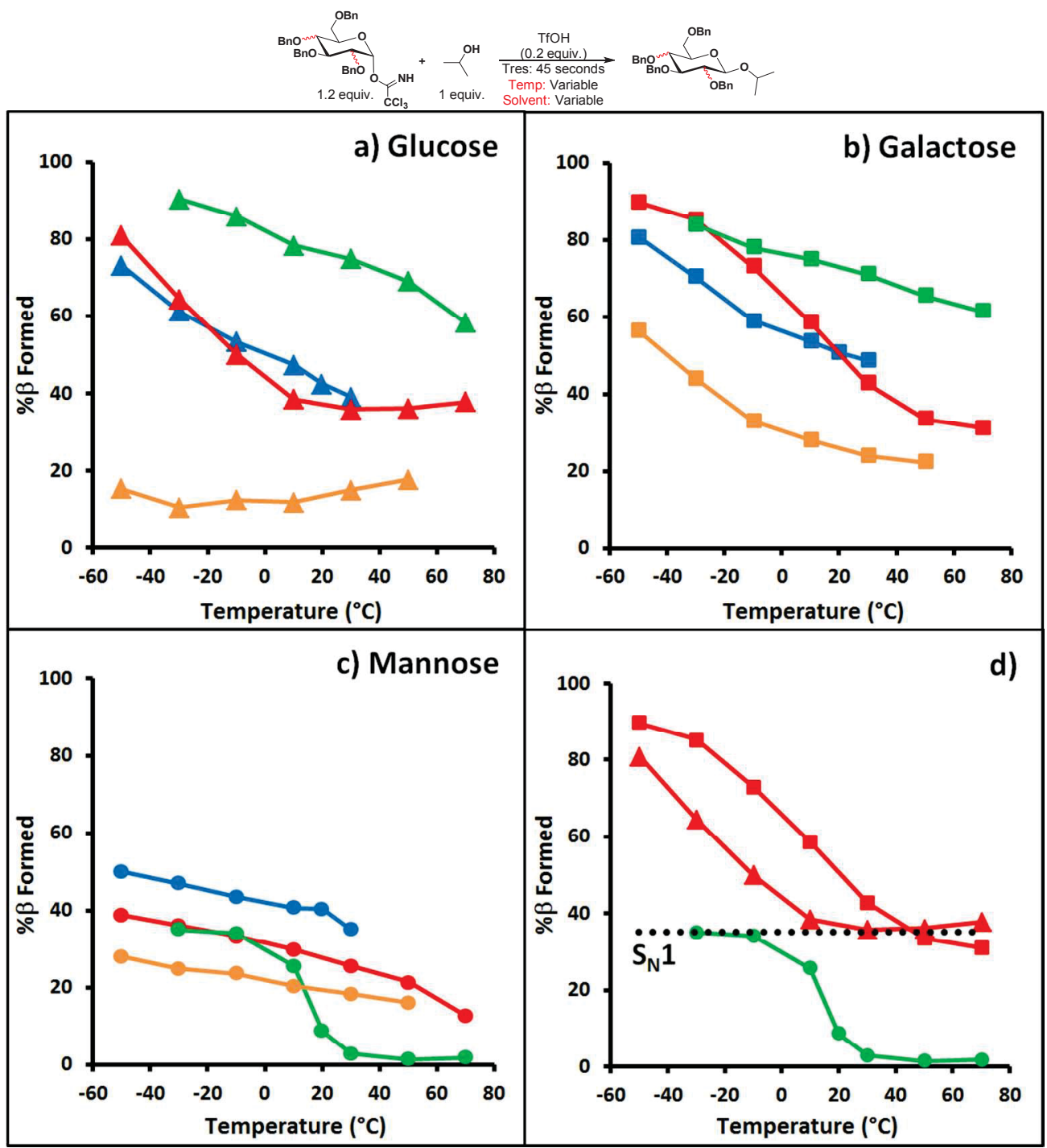

Figure 11: Comparison of four different solvents for the coupling of the three model donors with $i$ PrOH using TfOH as activator. For full experimental details, see SI. Figure code: Glucose ( $\mathbf{\Delta})$; Galactose (匹); Mannose (•); DCM (blue); Toluene (red); ACN (green); MTBE (orange).

Discussion:

The multitude of factors that influence the stereoselectivity of glycosylations has traditionally been challenging to identify and disentangle. Reproducibility issues due to the sensitivity of glycosylations to environmental factors compounded the problem. As such, while some aspects of glycosylations have been studied in great depth, the interrelationship of these factors and their degree of influence has remained unclear. Here, we provide the most complete picture to date of what these influencing factors are, what their affect is, and a direct comparison of how the degrees of influence of these factors rank with respect to one another (Figure 12).

The most important lesson this work teaches us is that the donor and acceptor coupling partners possess inherent preferences for the formation of either the $\alpha$ - or $\beta$-stereoisomer, and that these preferences can be enhanced, diminished, or overridden by environmental variables. The first, and most important, of the variables dictated by coupling partners is the stereochemistry of the $\mathrm{C} 2$ position of the donor, acting as a mechanistic divergent point in the coupling. Mannose, bearing a $\mathrm{C} 2$ axial ether, has a strong inherent preference for the formation of the $\alpha$-product. The 
degree of mannose's $\alpha$-selectivity can be enhanced in a number of ways, most notably by choice of solvent and temperature. Solvents capable of stabilizing cationic intermediates such as the $\pi$ system of toluene or the lone pairs of MTBE or ACN increase the amount of alpha product formed, as does an increase in temperature, though to a lesser extent. The next most influential factor for mannose couplings is the activator, where the nature of the activator's conjugate base can change the selectivity at higher temperatures. Least influential appears to the acceptor, where minor differences in sterics and electronics appear to have a correspondingly minor impact on selectivity. The exception is poorly nucleophilic acceptors such as fluorinated alcohols, whose strong electron withdrawing groups give highly $\alpha$-selective mannosylations. Finally, the mannose donor is highly sensitive to water, with sub-stoichiometric amounts of water being sufficient to negate the environmental effects described above.

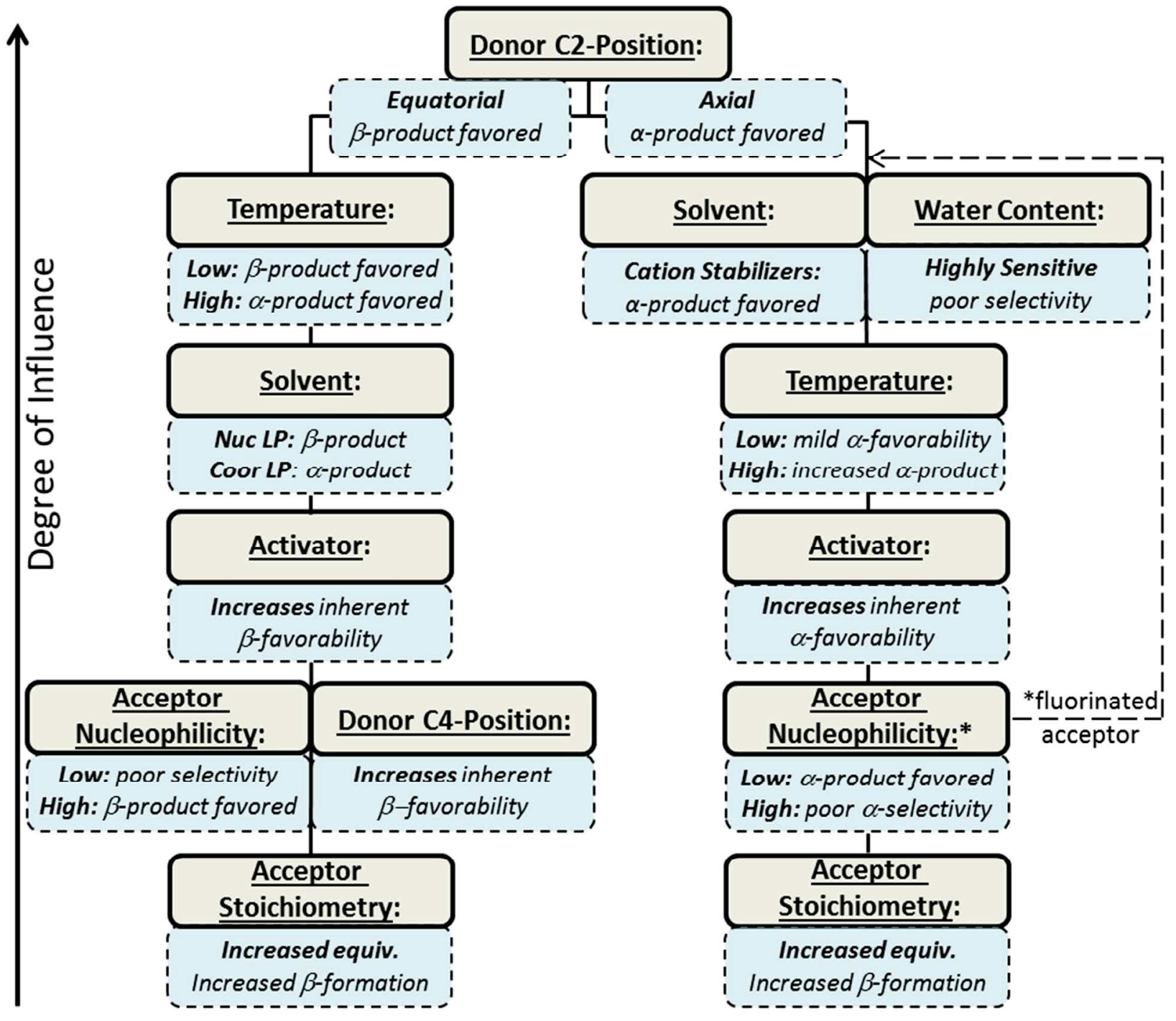

Figure 12: Degree of influence of environmental variables and permanent factors on the stereoselective outcome of glycosylation.

Donors lacking an axial C2-ether, such as glucose and galactose, have an inherent preference for the formation of the $\beta$-stereoisomer. However, this selectivity is more easily influenced than what is observed for the mannose branch. The glucose/galactose class of glycosylations is strongly influenced by the temperature, favoring the $\beta$-product at lower 
temperatures and a weak preference for alpha at higher temperatures. The next most influential factor is solvent, where non-halogen lone pair electrons play a key role: the nucleophilic acetonitrile enhancing $\beta$-isomer formation, while the coordinating MTBE enhancing the formation of the $\alpha$-isomer. Similar to mannose, the activator enhances the inherent selectivity preference of the donor, here the $\beta$-product. The degree of influence of the stereochemistry of the $\mathrm{C} 4$ position is similar to that of the acceptor's nucleophilicity. When the $\mathrm{C} 4$ ether is axial (galactose), a moderate increase in the $\beta$-selectivity is observed, as is the case with stepwise increases in the nucleophilicity of the acceptor. Poor nucleophiles, such as fluorinated alcohols, are poorly selective, weakly favoring the $\alpha$-stereoisomer. Finally, glucose/galactose glycosylations can be slightly more $\beta$-selective with increased equivalents of acceptor. This branch of couplings appears to be significantly less sensitive to water than mannosylations.

While the glycosylation coupling partners, in particular the donor, dictate the favored stereochemical outcome, these inherent preferences can be manipulated by judicious choice of environmental conditions. The selectivity is predominantly determined by the set of intermediates formed following decomposition of the activated donor. The nature, ratio, and stability of these undetermined structures is influenced both by the stereoelectronics of the donor and, primarily, by the temperature, solvent, and conjugate base of the acceptor. The environmental conditions can also be utilized to influence the mechanistic pathway followed, increasing either the $\mathrm{S}_{\mathrm{N}} 1$ or $\mathrm{S}_{\mathrm{N}}$ 2-character of the glycosylation. Glucose is the donor most susceptible to manipulation of the stereochemical outcome, followed by galactose, and mannose.

Based on this work, it should be possible to identify conditions to maximize the proportion of either the $\alpha$ - or $\beta$-product for a given coupling pair. The coupling of the benzylated glucosyl $\alpha$-trichloroacetimidate with isopropanol serves to showcase this ability (Figure 13). In DCM using $\mathrm{Tf}_{2} \mathrm{NH}, 11: 1$ selectivity favoring the $\beta$-diastereomer is observed. When the reaction is run in MTBE with $\mathrm{TfOH}$ as activator at $-30{ }^{\circ} \mathrm{C}$, the selectivity is reversed to give a 9:1 ratio favoring the $\alpha$-diastereomer.

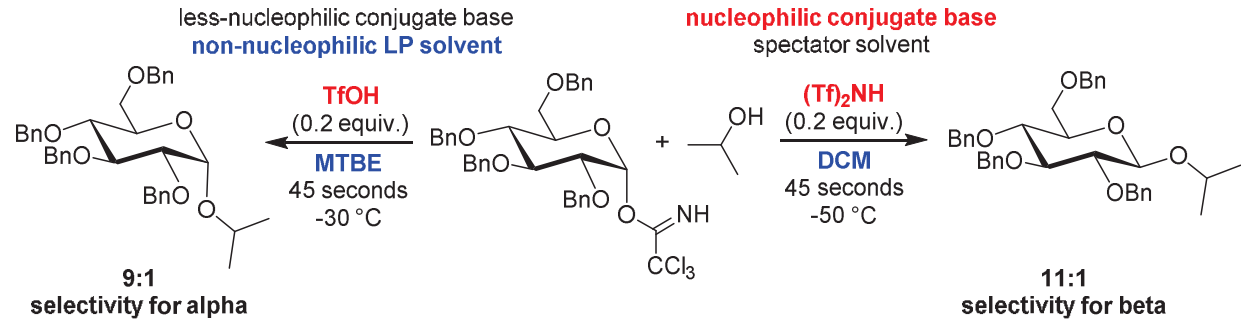

Figure 13: Tunable stereoselective outcome ratios of a given glycosylation coupling pair based on variation of environmental conditions.

In conclusion, an in depth-analysis of factors that influence the outcome of glycosylation reactions was possible thanks to an instrument that enables the systematic execution of reactions quickly, reliably, and reproducibly. The results of these automated flow experiments were used to identify and interrogate both permanent and environmental variables that influence the stereoselectivity. For the first time, the extent that these variables affect the outcome of glycosylations was quantified, and directly compared. As a result, an empirical understanding of how to control the stereochemical outcome of a glycosylation was gained. Further studies quantifying the degree of dominance of these interdependent variables, exploring the competing mechanistic pathways, and the modelling of these complex systems are currently underway.

\section{Acknowledgements:}


The authors would like to thank generous financial support provided by the Max Planck Society and DFG InCHeM (FOR 2177). We are also sincerely thankful to Dr. Fei-Fei Xu, Dr. Marilda P. Lisboa, Dr. Ju Yuel Baek, Eva Settels, and Olaf Niemeyer (all MPIKG) for their suggestions and support.

\section{Corresponding Authors: \\ kerry.gilmore@mpikg.mpg.de \\ peter.seeberger@mpikg.mpg.de \\ These authors contributed equally to this work}

\section{Supporting Information:}

Detailed experimental procedures, complete datasets, additional graphs and control studies, details regarding automation and instrumentation, as well as characterization data. This information is available free of charge at http://pubs.acs.org.

\section{References:}

${ }^{1}$ (a) A. Varki, Glycobiology, 1993, 3, 97-130. (b) R. A. Dwek, Chem. Rev., 1996, 96, 683-720.

${ }^{2}$ Hofmann, J.; Hahm, H. S.; Seeberger, P. H.; Pagel, K. Nature 2015, 526, 241-244.

3 a) Crich, D. Acc. Chem. Res. 2010, 43, 1144-1153; b) Bohé, L.; Crich, D. Carbohyd. Res. 2015, 403, 48-59.

${ }^{4}$ Ranade, S. C.; Demchenko, A. V. J. Carbohyd. Chem. 2013, 32, 1-43.

${ }^{5}$ Hosoya, T.; Takano, T.; Kosma, P.; Rosenau, T. J. Org. Chem. 2014, 79, 7889-7894.

${ }^{6}$ Tvaroska, I.; Bleha, T.; Adv. Carbohydr. Chem. Biochem. 1989, 47, 45-123.

${ }^{7}$ Cumpstey, I. Org. Biomol. Chem. 2012, 10, 2503-2508.

${ }^{8}$ Frihed, T. G.; Bols, M.; Pedersen, C. M. Chem. Rev. 2015, 115, 4963-5013.

${ }^{9}$ Park, J.; Kawatkar, S.; Kim, J.-H.; Boons, G.-J. Org. Lett. 2007, 9, 1959-1962.

${ }^{10}$ Reports discuss the observation of $\alpha$-triflates and cationic intermediates under extreme conditions, see: a) Crich, D. J. Org. Chem. 2011, 76, 9193-9209; b) Martin, A.; Arda, A.; Désiré, J.; Martin-Mingot, A.; Probst, N.; Sinaÿ, P.; Jiménez-Barbero, J.; Thibaudeau, S.; Blériot, Y. Nature Chem. 2016, 8, 186-191.

${ }^{11}$ Satoh, H.; Manabe, S. Chem. Soc. Rev. 2013, 42, 4297-4309.

12 a) Nigudkar, S. S.; Demchenko, A. V. Chem. Sci. 2015, 6, 2687-2704; b) Garcia, A.; Otte, D. A. L.; Salamant, W. A.; Sanzone, J. R.; Woerpel, K. A. Angew. Chem. Int. Ed. 2015, 54, 3061-3064; c) Lourenço, E. C.; Ventura, M. R. Tetrahedron 2013, 69, 7090-7097.

${ }^{13}$ a) Alabugin, I. V.; Gilmore, K. M.; Peterson, P. W. WIREs Comp. Mol. Sci. 2011, 1, 109-141; b) Alabugin, I. V. Stereoelectronic Effects: A Bridge Between Structure and Reactivity. (2016) John Wiley \& Sons.

${ }^{14}$ Transient protecting groups can also be used to gain stereoselectivity through sterically blocking one face, see: Hoang, K. L. M.; He, J.-x.; Báti, G.; Chan-Park, M. B.; Liu, X.-W. Nature Commun. 2017, 8, 1146.

${ }^{15}$ a) Kalikanda, J.; Li, Z. J. Org. Chem. 2011, 76, 5207-5218; b) Baek, J. Y.; Lee, B.-Y.; Jo, M. G.; Kim, K. S. J. Am. Chem. Soc. 2009, 131, 17705-17713.

${ }^{16}$ Fraser-Reid, B.; Wu, Z.; Udodong, U. E.; Ottosson, H. J. Org. Chem.1990, 55, 6068-6070.

${ }^{17}$ Jensen, H. H.; Nordstrom, L. U.; Bols, M. J. Am. Chem. Soc. 2004, 126, 9205-0213.

18 a) Huang, M.; Garrett, G. E.; Birlirakis, N.; Bohé, L.; Pratt, D. A.; Crich, D. Nat. Chem. 2012, 4, 663-667; b) Crich, D.; Cai, W. J. Org. Chem. 1999, 64, 4926-4930; c) Crich, D.; Chandrasekera, N. S. Angew. Chem. Int. Ed. 2004, 43, 5386-5389.

${ }^{19}$ Trichloracetimidates: a) Schmidt, R. R.; Michel, J. Angew. Chem. Int. Ed. 1980, 19, 731-733; thioglycosides: b) Pozsgay, V.; Jennings, H. J. Tetrahedron Letters 1987, 28, 1375-1376; c) Lemieux, R. U. Can. J. Chem. 1951, 29, 1079-1091; d) Garegg, P. J. Adv. Carbohyd. Chem. Bi. 1997, 52, 179-205; phosphates/phosphites: e) Hashimoto, S.; Honda, T.; Ikegami, S. J. Chem Soc., Chem. Commun. 1989, 685-687; f) Martin, T. J.; Schmidt, R. R. Tetrahedron Letters 1992, 33, 6123-6126; g) Martin, T. J.; Brescello, R.; Toepfer, A.; Schmidt, R. R. Glyconjugate Journal 1993, 10, 16-25; h) Kondo, H.; Ichikawa, Y.; Wong, C.-H. J. Am. Chem. Soc. 1992, 114, 8748-8750; i) Sim, M. M.; Kondo, H.; Wong, C.-H. J. Am. Chem. Soc. 1993, 115, 2260-2267; Overviews: j) Demchenko, A. V. Curr. Org. Chem. 2003, 7, 35-79; k) Demchenko, A. V. Synlett 2003, 1225-1240.

${ }^{20}$ a) Paulsen, H. Angew. Chem. Int. Ed. 1982, 21, 155-173; b) Sinay, P. Pure Appl. Chem. 1978, 50, 1437-1452. 
${ }^{21}$ Orgueira, H. A.; Bartolozzi, A.; Schell, P.; Seeberger, P. H. Angew. Chem. Int. Ed. 2002, 41, 2128-2131.

${ }^{22}$ (a) van der Vorm, S.; Hansen, T.; Overkleeft, H. S.; van der Marel, A.; Codée, J. D. C. Chem. Sci. 2017, 8, 18671875; (b) Schumann, B.; Parameswarappa, S. G.; Lisboa, M. P.; Kottari, N.; Guidetti, F.; Pereira, C. L.; Seeberger, P. H. Angew. Chem. Int. Ed. 2016, 55, 14431-14434.

${ }^{23}$ Hosoya, T.; Kosma, P.; Rosenau, T. Carbohyd. Res. 2015, 401, 127-131.

${ }^{24}$ Kononov, L. O.; Malysheva, N. N.; Orlova, A. V.; Zinin, A. I.; Laptinskaya, T. V.; Kononova, E. G.; Kolotyrkina, N. G. Eur. J. Org. Chem. 2012, 1926-1934.

${ }^{25}$ a) Huang, M.; Retailleau, P.; Bohé, L.; Crich, D. J. Am. Chem. Soc. 2012, 134, 14746-14749; b) Adero, P. O.; Furukawa, T.; Huang, M.; Mukherjee, D.; Retailleau, P.; Bohé, L; Crich, D. J. Am. Chem. Soc. 2015, 137, 1033610345.

${ }^{26}$ Tanaka, K.; Mori, Y.; Fukase, K. J. Carbohdr. Chem. 2009, 28, 1-11.

${ }^{27}$ Plutschack, M. B.; Pieber, B.; Gilmore, K.; Seeberger, P. H., Chem. Rev. 2017, 117, 11796-11893.

${ }^{28}$ Ratner, D. M.; Murphy, E. R.; Jhunjhunwala, M.; Snyder, D. A.; Jensen, K. F.; Seeberger, P. H. Chem. Commun. 2005, 578-580.

${ }^{29}$ a) van der Vorm, S.; van Hengst, J. M. A.; Bakker, M.; Overkleeft, H. S.; van der Marel, G. A.; Codée, J. D. C. Angew. Chem. Int. Ed. 2018, DOI: 10.1002/anie.201802899; b) van der Vorm, S.; Overkleeft, H. S.; van der Marel, G.; D. C. Codée, J. J. Org. Chem. 2017, 82, 4793-4811.

${ }^{30}$ (a) Durantie, E.; Bucher, C.; Gilmour, R. Chem. Eur. J. 2012, 18, 8208; (b) Rencurosi, A.; Lay, L.; Russo, G.; Caneva, E.; Poletti, L. J. Org. Chem. 2005, 70, 7765.

${ }^{31}$ Further studies exploring the unique reactivies of the $\alpha$ - and $\beta$-donor will be discussed in a subsequent publication.

32 a) Yang, M. T.; Woerpel, K. A. J. Org. Chem. 2009, 74, 545-553; b) Chamberland, S.; Ziller, J. W.; Woerpel, K.

A. J. Am. Chem. Soc. 2005, 127, 5322-5323.

${ }^{33}$ Crich, D.; Vinogradova, O. J. Org. Chem. 2006, 71, 8473-8480.

${ }^{34}$ Lucero, C. G.; Woerpel, K. A. J. Org. Chem. 2006, 71, 2641-2647.

${ }^{35}$ Bucher, C.; Gilmour, R. Angew. Chem. Int. Ed. 2010, 49, 8724-8728.

${ }^{36}$ Kalikanda, J.; Li, Z. Carbohydr. Res. 2011, 346, 2380-2383.

${ }^{37} \alpha / \beta$-products of perbenylated glucose coupled to either 2,2,2-trifluoroethanol or 2,2-difluoroethanol could not be separated using the HPLC methods developed.

${ }^{38}$ A similar observation has been made for 2-deoxyglycosyl donors, see: Beaver, M. G.; Woerpel, K. A. J. Org. Chem. 2010, 75, 1107-1118.

${ }^{39}$ The concentration of the donor has been shown to influence the temperature of activation, the reaction time, and the yield, see: Kononov, L. O.; Fedina, K. G.; Orlova, A. V.; Kondakov, N. N.; Abronina, P. I.; Podvalnyy, N. M.; Chizhov, A. O. Carbohyd. Res. 2017, 437, 28-35.

${ }^{40}$ For a discussion about the nucleophilicity of the trifluoromethanesulfonate anion, see: Dhakal, B.; Bohé, L.; Crich, D. J. Org. Chem. 2017, 82, 9263-9269.

${ }^{41}$ Kowalska, K.; Pedersen, C. M. Chem. Commun. 2017, 53, 2040-2043.

${ }^{42}$ Kafle, A.; Liu, J.; Cui, L. Can. J. Chem. 2016, 94, 894-901.

${ }^{43}$ Satoh, H.; Hansen, H. S.; Manabe, S.; van Gunstersen, W. F.; Hünenberger, P. H. J. Chem. Theory Comput. 2010, 6, 1783-1797. 